\title{
Effectiveness of Montreal Cognitive Assessment for the diagnosis of mild cognitive impairment and mild Alzheimer's disease in Singapore
}

\author{
Amanda $\mathrm{Ng}^{1}$, MSc, Ivane ${\underline{C h e w^{1}}}$, BSocSc, Kaavya Narasimhalu ${ }^{2}$, PhD, Nagaendran Kandiah ${ }^{1,2}$, MRCP
}

INTRODUCTION Mild cognitive impairment $(\mathrm{MCl})$ is an important clinical entity with significant management implications. However, traditional screening tools lack the sensitivity needed to detect amnestic $\mathrm{MCl}(\mathrm{MCl}-\mathrm{A})$. Montreal Cognitive Assessment (MoCA) has yet to be validated for the diagnosis of $\mathrm{MCl}$ in a multiracial society such as Singapore. We thus aimed to study the effectiveness of MoCA for the diagnosis of $\mathrm{MCl}-\mathrm{A}$ in the Singapore population.

METHODS Data on patients with MCI-A and mild Alzheimer's disease (AD) was obtained from a prospectively collected clinical database between January 2008 and January 2011. Patients with no cognitive impairment (NCI) were recruited from among the spouses and friends of patients attending the memory clinic.

RESULTS There were a total of 212 participants (103 NCl, $49 \mathrm{MCl}-\mathrm{A}, 60$ mild AD). For the diagnosis of $\mathrm{MCl}-\mathrm{A}$, a MoCA score of $<26$ for patients with $\leq 10$ years of education, and a score of $<27$ for patients with $>10$ years of education provided a sensitivity of $>94 \%$. For the diagnosis of mild AD, a MoCA score of $<24$ for patients with $\leq 10$ years of education, and a score of $<25$ for patients with $>10$ years of education provided a sensitivity of $>85 \%$.

CONCLUSION In the Singapore population, we recommend cutoff scores of $26 / 27$ and $24 / 25$ be used to detect $\mathrm{MCl}-\mathrm{A}$ and mild $\mathrm{AD}$, respectively, when using MoCA. For patients with $\leq 10$ years of education, $\mathrm{a}+1$ point correction is needed.

Keywords: dementia, mild Alzheimer's disease, mild cognitive impairment, neuropsychological assessment

\section{INTRODUCTION}

Mild cognitive impairment $(\mathrm{MCl})$ is a heterogeneous clinical entity that spans between normal ageing and dementia in the elderly. ${ }^{(1)} \mathrm{MCl}$ is characterised as cognitive impairment(s) beyond normal ageing, with minimal or no decline in activities of daily living $(\mathrm{ADL}){ }^{(2)} \mathrm{MCl}$ represents a significant risk factor for dementia, with an annual conversion rate of about $10 \%-15 \%$ to mild Alzheimer's disease (AD). ${ }^{(3)}$ Hence, there are important management implications surrounding this clinical entity, including cognitive and pharmacological management. ${ }^{(4)}$ Early detection of $\mathrm{MCl}$ is crucial in order to allow optimum interventions as they become available, so as to reduce the risk of progression to dementia. Among the subtypes of $\mathrm{MCl}$, amnestic $\mathrm{MCl}(\mathrm{MCl}-\mathrm{A})$ is the most homogeneous and best characterised entity. It also has the highest rates of conversion to $\mathrm{AD}$.

Cognitive screening tools are useful for the clinical diagnosis of $\mathrm{MCl}$. While routine cognitive tools such as the mini-mental state examination (MMSE) have been demonstrated to be effective in the detection of dementia, these tools are less effective in the detection of $\mathrm{MCl}$, as most individuals with $\mathrm{MCl}$ score in the normal range on MMSE. ${ }^{(2,5)}$ The Montreal Cognitive Assessment (MoCA) was demonstrated to have good sensitivity and specificity for the detection of $\mathrm{MCl} .{ }^{(6)}$ However, due to cultural and language differences across regions, it is likely that different MoCA cutoff points would be required to aid the diagnosis of cognitive impairment in specific countries. For instance, Lee et al in 2008 demonstrated that a cutoff of 22/23 provided the highest sensitivity when diagnosing cognitive impairment in Korea, ${ }^{(7)}$ while Wen et al suggested that a cutoff of 26/27 was appropriate for a Chinese population. ${ }^{(8)}$ The original study reported a cutoff point of 25/26 in a Canadian population. ${ }^{(6)}$

In the present study, we aimed to determine the sensitivity and specificity of MoCA for the detection of MCl-A and mild AD in a multiracial population in Singapore. We hypothesised that a MoCA cutoff point of 26, similar to the original study by Nasreddine et $\mathrm{al}^{\left({ }^{(6)}\right.}$ would be applicable for diagnosing MCl-A in Singapore, while a MoCA score of 24-25 would be applicable for the diagnosis of mild AD.

\section{METHODS}

Data on $\mathrm{MCl}-\mathrm{A}$ and mild $\mathrm{AD}$ were obtained from a prospectively collected clinical database, which comprised patients with cognitive impairment managed at the National Neuroscience Institute, Singapore, between January 2008 and January 2011. Hospital-based controls with no cognitive impairment $(\mathrm{NCl})$ were recruited from among the spouses and friends of patients

Department of Neurology, National Neuroscience Institute, ${ }^{2}$ Duke-NUS Medical School, Singapore

Correspondence: Dr Nagaendran Kandiah, Consultant, Department of Neurology, National Neuroscience Institute, 11 Jalan Tan Tock Seng, Singapore 308433. Nagaendran_Kandiah@nni.com.sg 
Table I. Demographics and cognitive scores.

\begin{tabular}{|c|c|c|c|c|}
\hline Variable & $\mathrm{NCl}(\mathrm{n}=103)$ & MCl-A (n = 49) & Mild AD $(n=60)$ & p-value \\
\hline Age* (yrs) $^{*}$ & $56.35 \pm 8.27$ & $62.43 \pm 9.40$ & $72.58 \pm 7.15$ & $<0.001$ \\
\hline Male gender ${ }^{+}$ & $41(39.81)$ & $27(55.10)$ & $30(50.00)$ & 0.177 \\
\hline \multicolumn{5}{|l|}{ Ethnicity ${ }^{+}$} \\
\hline Chinese & $96(93.20)$ & $41(83.67)$ & $48(80.00)$ & 0.035 \\
\hline Malay & $2(1.94)$ & $1(2.04)$ & 2 (3.33) & 0.841 \\
\hline Indian & $2(1.94)$ & $4(8.16)$ & $5(8.33)$ & 0.117 \\
\hline Eurasian & $1(0.97)$ & $1(2.04)$ & $1(1.67)$ & 0.856 \\
\hline Other & $2(1.94)$ & $2(4.08)$ & $4(6.67)$ & 0.309 \\
\hline Education* (yrs) & $12.07 \pm 3.20$ & $10.93 \pm 4.28$ & $6.97 \pm 4.47$ & $<0.001$ \\
\hline MMSE* (graded out of 30 ) & $29(29.19 \pm 0.88)$ & $29(28.06 \pm 2.12)$ & $23(22.93 \pm 3.84)$ & 0.0001 \\
\hline MoCA $^{*}$ (graded out of 30$)$ & $29(28.56 \pm 1.45)$ & $28(27.00 \pm 3.02)$ & $21(20.80 \pm 4.06)$ & 0.0001 \\
\hline GDS* (graded out of 15 ) & $1(2.28 \pm 2.89)$ & $2(2.76 \pm 3.07)$ & $2(2.87 \pm 2.75)$ & 0.324 \\
\hline
\end{tabular}

*Data is expressed as mean \pm standard deviation. ${ }^{\dagger}$ Data is expressed as no. (\%). ${ }^{\ddagger}$ Data is expressed as median (mean \pm standard deviation).

AD: Alzheimer's disease; GDS: Geriatric Depression Scale; MCl-A: amnestic mild cognitive impairment; MMSE: mini-mental state examination; MoCA: Montreal Cognitive Assessment; $\mathrm{NCl}$ : no cognitive impairment

attending the memory clinic. Patients with significant symptoms of depression, scoring $>5$ on the modified Geriatric Depression Scale (GDS), were excluded from the study. The cognitive tests were performed on both the controls and patients by trained raters. The MMSE and MoCA (version 7.1) cognitive screening tests were used.(6,9) The 15-point GDS was used to exclude patients with significant depressive symptoms. ${ }^{(10)}$ For nonEnglish-speaking participants, the tests were translated to their native languages.

AD was diagnosed using the NINCDS-ADRDA (National Institute of Neurological and Communicative Disorders and Stroke-Alzheimer's Disease and Related Disorders Association) Alzheimer's Criteria, based on the history provided by the patients and caregivers. ${ }^{(11)}$ A Clinical Dementia Rating (CDR) of 1 represented mild AD. ${ }^{(12)} \mathrm{MCl}-\mathrm{A}$ was diagnosed using Petersen's criteria and defined by the presence of subjective memory concerns, impaired memory function for age and education, a CDR of 0.5 , preserved $\mathrm{ADL}$, and the absence of dementia. ${ }^{(2)}$

Corroborative history from a reliable caregiver was obtained for all patients with $\mathrm{MCl}-\mathrm{A}$. All patients in the present study did not fulfil the DSM-IV-TR (Diagnostic and Statistical Manual of Mental Disorders, 4th edition, text revision) criteria for dementia. Diagnoses of $\mathrm{MCl}-\mathrm{A}$ and mild $\mathrm{AD}$ were made by neurologists blinded to the patients' MoCA scores. Participants with $\mathrm{NCl}$ had a CDR score of 0 and an MMSE score of 27-30 (inclusive). $\mathrm{NCl}$ participants also had to be deemed cognitively normal based on an absence of significant impairment in cognitive functions or ADL following review by a clinician. Informed consent was obtained from all participants or their legal guardians before their data were included in the database, as per institutional ethics board requirements.

Demographic characteristics were compared across three groups ( $\mathrm{NCl}, \mathrm{MCl}-\mathrm{A}$ and mild $\mathrm{AD}$ ) using chi-square test for categorical data, one-way analysis of variance for continuous parametric data and Kruskal-Wallis test for continuous nonparametric data. Pairwise comparisons were performed using chi-square test for categorical variables, $t$-test for continuous parametric variables and Wilcoxon rank-sum test for continuous nonparametric variables. Ordinary least squares linear regression was performed to determine the relationship between age and education, and their effect on MoCA scores. Receiver operating characteristic (ROC) curve analysis was used to determine appropriate age- and education-adjusted MoCA cutoff scores for the detection of $\mathrm{MCl}-\mathrm{A}$ and mild AD. All statistical analyses were performed using Stata version 10.1 (StataCorp, College Station, TX, USA) software. All tests were two-tailed and conducted at the $5 \%$ level of significance.

\section{RESULTS}

Data from a total of 212 participants $(103 \mathrm{NCl}, 49 \mathrm{MCl}-\mathrm{A}, 60$ mild AD) were analysed. There was a fairly equal distribution of men and women across the three groups. The majority of the patients were of Chinese ethnicity, reflecting Singapore's cultural makeup. The mean age of the entire cohort was 62.35 years and the mean age of patients with mild AD was 72.58 years. Patients with mild AD had significantly lower education than those with $\mathrm{MCl}-\mathrm{A}$ and participants with $\mathrm{NCl}$ (Table I). NCl participants had a mean MMSE score of $29.19 \pm 0.88$ and a mean MoCA score of $28.56 \pm 1.45$. For patients with $\mathrm{MCl}-\mathrm{A}$, the mean MMSE and MoCA scores were $28.06 \pm 2.12$ and $27.00 \pm 3.02$, respectively. The mean MMSE and MoCA scores for patients with mild AD were $22.93 \pm 3.84$ and $20.80 \pm 4.06$, respectively. As shown in Table I, the mean MMSE and MoCA scores for the mild AD group were lower than the scores of patients with $\mathrm{MCl}-\mathrm{A}$, which were in turn lower than those of $\mathrm{NCl}$ participants.

As linear regression analysis revealed that age $(p<0.001)$ and education $(p<0.001)$ were independent factors influencing MoCA scores (Table II), ROC curve analyses were conducted to compare the $\mathrm{NCl}$ group against the $\mathrm{MCl}-\mathrm{A}$ group, and the $\mathrm{MCl}-\mathrm{A}$ group against the mild $\mathrm{AD}$ group, so as to determine optimal cutoff points for the two variables. The most appropriate cutoff point for education was found to be ten years, but there was no clear inflection point for age. Therefore, a decision was made to only apply cutoff points for 
Table II. Linear regression analysis of the effects of age and education on Montreal Cognitive Assessment scores.

\begin{tabular}{|c|c|c|c|c|c|c|c|c|}
\hline \multirow[t]{2}{*}{ Variable } & \multicolumn{4}{|c|}{ Univariate analysis } & \multicolumn{4}{|c|}{ Multivariate analysis } \\
\hline & Coefficient $\beta$ & $\mathbf{R}^{2}$ & $t$ & p-value & Coefficient $\beta$ & $\mathbf{R}^{2}$ & $t$ & p-value \\
\hline Age & -0.198 & 0.247 & -7.92 & $<0.001$ & -0.179 & 0.272 & -6.84 & $<0.001$ \\
\hline Education & 0.497 & 0.214 & 7.12 & $<0.001$ & 0.323 & 0.072 & 4.50 & $<0.001$ \\
\hline
\end{tabular}

Table III. Sensitivity and specificity of Montreal Cognitive Assessment cutoff scores, when stratified by years of education.

\begin{tabular}{|c|c|c|c|c|c|c|c|c|}
\hline \multirow{2}{*}{$\begin{array}{l}\text { Years of } \\
\text { education }\end{array}$} & \multicolumn{4}{|c|}{ MCI-A } & \multicolumn{4}{|c|}{ Mild AD } \\
\hline & Score & Sensitivity & Specificity & $\begin{array}{l}\text { Correctly } \\
\text { classified }\end{array}$ & Score & Sensitivity & Specificity & $\begin{array}{l}\text { Correctly } \\
\text { classified }\end{array}$ \\
\hline \multicolumn{9}{|l|}{$>10$} \\
\hline & $<26$ & 98.08 & 9.52 & 72.60 & $<24$ & 95.24 & 70.00 & 87.10 \\
\hline & $<27^{*}$ & 94.23 & 19.05 & 72.60 & $<25^{*}$ & 90.48 & 70.00 & 83.87 \\
\hline & $<28$ & 82.69 & 28.57 & 67.12 & $<26$ & 90.48 & 90.00 & 90.32 \\
\hline & $<25$ & 96.00 & 25.00 & 75.71 & $<23$ & 85.00 & 72.22 & 76.79 \\
\hline & $<26^{*}$ & 96.00 & 30.00 & 77.14 & $<24^{*}$ & 85.00 & 80.56 & 82.14 \\
\hline & $<27$ & 88.00 & 40.00 & 74.29 & $<25$ & 75.00 & 80.56 & 78.57 \\
\hline
\end{tabular}

All data except for Score are presented as percentage. ${ }^{*}$ Chosen cutoff scores. AD: Alzheimer's disease; MCl-A: amnestic mild cognitive impairment

education. At the cutoff limit of ten years of education, we found that the sensitivity of MoCA for the differentiation of MCl-A from mild AD dropped drastically from $72.09 \%$ to $48.84 \%$. Similarly, for the differential diagnosis of $\mathrm{NCl}$ from $\mathrm{MCl}-\mathrm{A}$, the sensitivity of MoCA dropped from $88.35 \%$ to $50.49 \%$.

Repeat ROC analyses after correction for education demonstrated that a MoCA cutoff score of $<24$ for the detection of mild $A D$, when years of education $\leq 10$, had a good sensitivity of $85.00 \%$ and a specificity of $80.56 \%$. When years of education $>10$, a cutoff score of $<25$ was found to give a sensitivity of $90.48 \%$ and a specificity of $70.00 \%$ for the differentiation of mild AD (Table III).

\section{DISCUSSION}

Our findings suggest that a MoCA cutoff score of 26/27 differentiates between $\mathrm{NCl}$ and $\mathrm{MCl}-\mathrm{A}$, while a cutoff score of 24/25 differentiates between MCl-A and mild AD in the Singapore population, with a correction of +1 point when the patient has $\leq 10$ years of education. Cutoff scores were chosen based on their balance of sensitivity and specificity. Based on the above criteria, a MoCA cutoff score of $<24$ should have been chosen for detecting mild $A D$ in patients with more than 10 years of education, as it had a sensitivity of $95.24 \%$ and specificity of $70 \%$. However, we opted to use a cutoff point of $<25$, which also had a high sensitivity $(90.48 \%$ ), so that the general rule of adding 1 point to the total MoCA score for patients with more than 10 years of education could be applied to both groups. This was to facilitate administration and diagnoses in clinical settings.

Previous studies validating MoCA have only established single cutoff points - the original cutoff was 25/26 for the detection of cognitive impairment, ${ }^{(6)} 25 / 26$ for the detection of $\mathrm{MCl}$ using the Japanese version of MoCA, ${ }^{(13)}$ and 23/24 for the detection of cognitive impairment in a community- based cohort in southeastern United States. ${ }^{(14)}$ Our study established education-adjusted MoCA cutoff points for the detection of $\mathrm{MCl}-\mathrm{A}$ and mild $\mathrm{AD}$ as this could assist clinicians in dissociating the two conditions, which tend to have a similar clinical presentation. Although the cutoff point used for years of education in the original study on MoCA was 12 years, ${ }^{(6)}$ we found that a 1-point correction was needed in patients with less than 10 years of education in our study. This change in cutoff score from the original study was effected due to the education system in Singapore, where the average population spends 10 years in school to obtain a basic education. The cutoff scores used in our study differ from the scores determined in the original MoCA study, as well as from other similar studies elsewhere, highlighting the importance of conducting population-specific validations of MoCA in order to maintain its effectiveness as a screening tool.

There were strengths and limitations to this study. Among its strengths are the relatively large sample size of patients, the use of trained raters, and the novelty of establishing two separate cutoff scores for $\mathrm{MCl}-\mathrm{A}$ and mild $\mathrm{AD}$, as opposed to a single cutoff score for both conditions. However, the study's retrospective design was a limitation. Additionally, as patients were recruited from a tertiary hospital, generalisation of our results to the larger community will need to be performed with caution.

In conclusion, we recommend that MoCA cutoff scores of 26/27 and 24/25 be used to detect MCI-A and mild AD, respectively, in the Singapore population. An added 1-point correction will be needed for patients with 10 years of education or less.

\section{ACKNOWLEDGEMENTS}

We would like to thank all clinicians at the Department of Neurology, National Neuroscience Institute, Singapore, for providing data on patients with $\mathrm{MCl}-\mathrm{A}$ and mild AD. 


\section{REFERENCES}

1. Chertkow $\mathrm{H}$, Massoud $\mathrm{F}, \mathrm{Nasreddine} Z$, et al. Diagnosis and treatment of dementia: 3. Mild cognitive impairment and cognitive impairment without dementia. CMAJ 2008; 178:1273-85.

2. Petersen RC, Smith GE, Waring SC, et al. Mild cognitive impairment: clinical characterization and outcome. Arch Neurol 1999; 56:303-8.

3. Grundman M, Petersen RC, Ferris $\mathrm{SH}$, et al. Mild cognitive impairment can be distinguished from Alzheimer disease and normal aging for clinical trials. Arch Neurol 2004; 61:59-66.

4. Sherwin BB. Mild cognitive impairment: potential pharmacological treatment options. J Am Geriatr Soc 2000; 48:431-41.

5. Wind AW, Schellevis FG, Van Staveren G, et al. Limitations of the MiniMental State Examination in diagnosing dementia in general practice. Int J Geriatr Psychiatry 1997; 12:101-8.

6. Nasreddine ZS, Phillips NA, Bédirian V, et al. The Montreal Cognitive Assessment, MoCA: a brief screening tool for mild cognitive impairment. J Am Geriatr Soc 2005; 53:695-9.

7. Lee JY, Lee DW, Cho SJ, et al. Brief screening for mild cognitive impairment in elderly outpatient clinic: validation of the Korean version of the Montreal Cognitive Assessment. J Geriatr Psychiatry Neurol 2008; 21:104-10.
8. Wen HB, Zhang ZX, Niu FS, Li L. [The application of Montreal cognitive assessment in urban Chinese residents of Beijing]. Zhonghua Nei Ke Za Zhi 2008; 47:36-9. Chinese.

9. Folstein MF, Folstein SE, McHugh PR. "Mini-mental state". A practical method for grading the cognitive state of patients for the clinician". J Psychiatr Res 1975; 12:189-98.

10. Sheikh JI, Yesavage JA. Geriatric Depression Scale (GDS): recent evidence and development of a shorter version. Clin Gerontol 1986; 5:165-73.

11. McKhann G, Drachman D, Folstein M, et al. Clinical diagnosis of Alzheimer's disease: report of the NINCDS-ADRDA Work Group under the auspices of Department of Health and Human Services Task Force on Alzheimer's Disease. Neurology 1984; 34:939-44.

12. Morris JC. The Clinical Dementia Rating (CDR): current version and scoring rules. Neurology 1993; 43:2412-4.

13. Fujiwara Y, Suzuki H, Yasunaga M, et al. Brief screening tool for mild cognitive impairment in older Japanese: validation of the Japanese version of the Montreal Cognitive Assessment. Geriatr Gerontol Int 2010; 10:225-32.

14. Luis CA, Keegan AP, Mullan M. Cross validation of the Montreal Cognitive Assessment in community dwelling older adults residing in the Southeastern US. Int J Geriatr Psychiatry 2009; 24:197-201. 